\title{
ANALYSIS OF IMPACT OF THE DIFFERENT NODE ARCHITECTURES AND WAVELENGTH CHANNEL PROTECTION ON THE AVAILABILITY WDM RING NETWORKS
}

This paper investigates the availability of WDM (Wavelength Division Multiplying) ring networks. An analysis of different nodes, composed of passive and active components, is then carried out, as well as their availability. The availability for optical link, and especially the Span Protection and Route Diversity, is analysed as well. The wavelength channel protection principles being used in WDM ring networks are particularly explicated: OCh (Optical Channel) dedicated and OCh shared protection. The availability models for a WDM ring using these types of protection are given as well as the impact of availability for nodes and optical links on the entire ring availability. Data on the intensity of failures and mean time to repair are taken from different literature.

Key words: WDM, wavelength channel, availability, protection.

\section{Introduction}

The availability of services is an important issue in today's optical networks. The increase in data transmission and development of Internet traffic require the high capacity transmission systems. Thanks to the development of fotonic technologies, systems are designed based on Wavelength Division Multiplexing (WDM) having high transmission speeds by a single fiber. In those networks, the service interrupt for whatever reason, caused by equipment failure, or by human factor, can cause unavailability of communication services, as well as heavy losses to the users and network operators. Therefore, the application of protection in such systems has important role for the network operators [1]. Contracts between the operators and their users are always made on the Service Level Agreement (SLA) which is very strict, as far as availability and service quality are concerned. The possibility to ensure high availability is the key one for the operators to maintain the existing and to aquire new users; this mostly reflecting in the price of their network.

The operators may largely invest to equip their network with the high quality of hardware [2]. However, the quality improvement of these components beyond the present level for commercial systems is hard to achieve, and it is beyond their control. More acceptable way to ensure high availability is the use of different protection strategies. Although there are more protection strategies we shall concentrate in this work at the wavelength channel protection of WDM which is very similar to the path protection of SDH (Synchronous Digital Hierarchy) technology [3].
In this work we are going to analyze the availability between two WDM ring terminals using the wavelength channel protection: - OCh Dedicated protection,

- OCh Shared protection.

This work is finding out what is the impact of a failure on optical cables and equipment on the availability of connection. The models and analysis of different node structures are given as well as their impact on availability and the availability of node depending on the manner it is being used in the network (add, drop, passthrough). In this work we focus on the wavelength channel protection strategy, which consists of ensuring the protection channel for each working channel on the end to end basis.

\section{Availability theory}

Availability A in some system is the probability that it will be functional in a given time, or it is the time relation in which the system is correct in relation to the total time. Unavailability $U$ of a system is the probability complementary to availability. At reporting about system performances, the unavailability is often expressed as a Mean Down Time (MDT) in minutes of the year or:

$$
M D T=\frac{M T T R}{M T T R+M T T F}
$$

Where MTTF is Mean Time to Failure, and MTTR is Mean Time to Repair [4]. In this work we suppose that MTTF for the wavelength channel is constant and independent on component

\footnotetext{
* Ivan Rados ${ }^{1}$, Ladislav Schwartz ${ }^{2}$

${ }^{1}$ Department of Transmission Systems HT d.d., Mostar, Bosnia and Herzegovina,

${ }^{2}$ Faculty of Electrical Engineering, University of Zilina, Slovakia, E-mail ladislav.schwartz@fel.uniza.sk,
} 
ageing. $M T T F=1 / \lambda$, where $\lambda$ is the intensity of failure usually expressed in FIT-a (Failure in Time), 1FIT $=1$ failure in hours.

The basic transport entity of WDM systems is a wavelength channel. This is the simplex type of connection with a standard of value between two nodes (for example, $2.5 \mathrm{~Gb} / \mathrm{s}$ ). The wavelength channel in an unprotected system (serial structure) generally passes through nodes which can be optical add/drop multiplexers, optical switches and optical links. The availability of a serial structure equals to the availability product of individual components, namely:

$$
A_{s}=\prod_{i=1}^{n} A_{i}
$$

This structure is unable to restore the communication between two nodes in the case of a failure on any of components or the connection is functional if and only if all components of the structure are correct. A substantial availability improvement can be obtained by the application of a parallel structure, which means that in addition to the working path there is a protection one, too. It is certainly important that these two paths are completely independent or that they do not have common components [5]. If we characterize the availabilities of working and protection path as the two completely independent elements ( $w$ and $p$ ), then the availability of such a structure is the union of two non-disjunctive events or the events which do not exclude each other because there is no complementary variable.

$$
A_{p}=P(w \cup p)
$$

In order that these two events be mutually exclusive it is necessary to transform this union of non-disjunctive events into a union of disjunctive events in which the complementary variables show up. By a simple analysis using De Morgan's laws we obtain that the availability is

$$
A=P|w \cup(\bar{w} \cap p)|
$$
section

By using the distributive law with regard to the union and

$$
A \cup(B \cap C)=(A \cup B) \cap(A \cup C)
$$

we obtain that

$$
A_{p}=P(w \cup \bar{w}) \cap(w \cup p)
$$

Since $w \cup \bar{w}=1$ (full set) we can write

$$
A_{p}=P[1 \cap(w \cup p)]
$$

and to the identity $1 \cap p=p$, so is

$$
A_{p}=P(w \cup p)
$$

And this is actually the availability of the parallel structure. The connection availability for a wavelength channel is [7].

$$
A_{p}=A_{k}=A_{w}+A_{p}-A_{w} A_{p}
$$

The connection between two nodes in a parallel structure will be incorrect if, and only if there is a failure on the working and protection path. Of course, the connection can not be established if a failure occurs in terminal nodes because they are the common components of the working and protection path.

\section{Availability of the link and different node architectures}

Since the nodes are generally connected by optical links, irrespective of their architecture, in this section we shall analyze the availability for links and different node architectures.

\subsection{Link availability}

In order to determine the wavelength channel availability between two nodes we shall introduce some terms relative to the availability of the node and the optical link connecting two nodes [6].

- $a_{O L_{i}}$, availability of i-th link on working path,

- $-a_{n_{i}}$, availability of $j$-the node which belongs to the working path.

An unprotected link consists of the optical fiber, optical booster $\left(a_{B O A}\right)$, amplifier $\left(a_{O A}\right)$, line amplifier $\left(a_{L O A}\right)$ and optical pre-amplifier $\left(a_{O P A}\right)$. In order that the connection will be functional, all components optical link must be correct. The most often cause of an optical link failure is the breakdown of optical fiber. Since, in the case of the cable breakdown, mostly all the fibers break, we shall suppose that the failures of fiber and cable are fully dependent. Therefore, instead of the availability for fiber, we shall take the availability for cable $\left(a_{O C}\right)$.

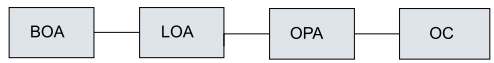

Fig. 1 Unprotected link structure

The availability of the link without the protection equals to the availability product of individual components.

$$
a_{O L N P_{i}}=a_{B O A} \times a_{L O A} \times a_{O P A} \times a_{O C}
$$

In the second instance shown in figure 2, two nodes are connected by two fibers belonging to the same cable.

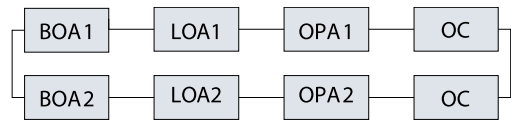

Fig. 2 Span protected link 
The availability for this structure (Span Protection-SP) equals to the parallel addition of the individual branch availabilities. So it can be written:

$$
\begin{aligned}
a_{O L . S P} & =a_{w} \cup a_{p}=a_{w}+a_{p}-a_{w} \cap a_{p} \\
& =a_{B O A 1} a_{L O A 1} a_{P O A 1} a_{O C}+a_{B O A 2} a_{L O A 2} a_{O P A 2} a_{O C}- \\
& -\left(a_{B O A 1} a_{B O A 2} a_{L O A 1} a_{L O A 2} a_{O P A 1} a_{O P A 2} a_{O C} a_{O C}\right)
\end{aligned}
$$

Although in the above equation's small brackets we have a product of two equal members $a_{O C} \times a_{O C}$, only one is present in the formula for availability $a_{O C}$ because the cause of node failure is the same so we get:

$$
\begin{aligned}
a_{O L ., S P} & =a_{B O A 1} a_{L O A l_{1}} a_{P O A 1} a_{O C}+a_{B O A 2} a_{L O A 2} a_{O P A 2} a_{O C}- \\
& -\left(a_{B O A 1} a_{B O A 2} a_{L O A 1} a_{L O A 2} a_{O P A 1} a_{O P A 2} a_{O C}\right)
\end{aligned}
$$

In this type of protection it is actually the protection in the case of a failure on some of the amplifiers but not the optical fibers.

In the third type of protection (route diversity-RD), the link structure is same as in span protection, in that the nodes are connected by optical fibers belonging to completely separated cables, as shown in figure 3 .

$$
\begin{aligned}
& a_{O L, R D}=a_{B O A 1} a_{L O A 1} a_{P O A 1} a_{O C 1}+a_{B O A 2} a_{L O A 2} a_{O P A 2} a_{O C 2}- \\
& -\left(a_{B O A 1} a_{B O A 2} a_{L O A 1} a_{L O A 2} a_{O P A 1} a_{O P A 2} a_{O C 1} a_{O C 2}\right)
\end{aligned}
$$

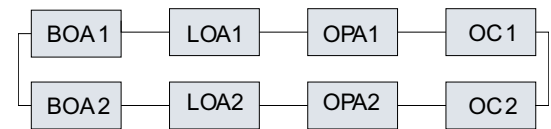

Fig. 3 Completely separated links

This type of protection protects the connection in the case of a failure on transmission equipment as well as optical cables.

\subsection{Availability for different node structure}

Nodes in which a wavelength channel is being dropped and added are called termination nodes. Nodes through which a wavelength channel runs from "west" to "east" and are located between terminal nodes are called pass-through nodes. Since the wavelength channel passes through the different components inside these two types of nodes their availability is different.

- $-a_{n_{i}}$, terminal node is a working one

- $-a_{n_{j}}$, pass-through node is working one

If all nodes of the same type are the same we have:

$a_{n_{i} t}=a_{n t}, \forall j$,

$a_{n_{j} p}=a_{n p}, \forall j$

\subsubsection{Node with passive components}

If passive components are used, the node structure in the $1+1$ wavelength channel protection are show in figure 4

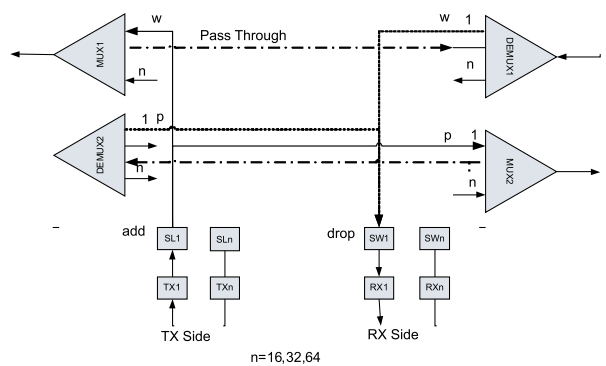

Fig. 4 Node structure for $1+1$ protection

For the instance of a terminal node (bidirectional communication), the wavelength channel passes through the transmitter, divider, multiplexers, demultiplexers, switch and receiver, so that the terminal node availability for one wavelength channel is

$$
\begin{gathered}
a_{n t, 1+1(1)}=a_{T X 1} \times a_{S P L 1} \times\left(a_{M U X 1} \cup a_{M U X 2}\right) \times \\
\left(a_{D M U X 1} \cup a_{D M U X 2}\right) \times a_{S W 1} \times a_{R X 1}
\end{gathered}
$$

In pass through nodes, a wavelength channel passes through the demultiplexers and multiplexers so the availability for passthrough nodes is:

$$
a_{n p, 1+1(1)}=a_{M U X 1} \times a_{D M U X 1} \times a_{M U X 2} \times a_{D M U X 2}
$$

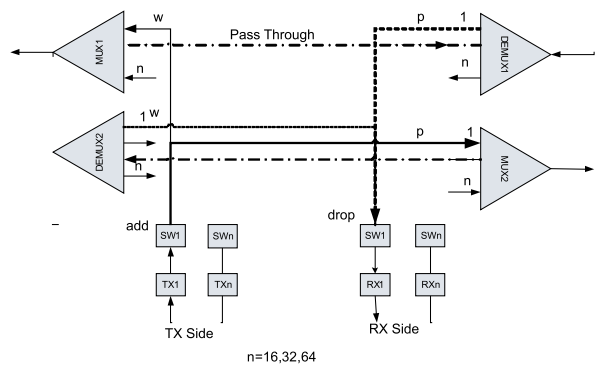

Fig. 5 Node structure for 1:1 protection

In $1: 1$ protection, if passive components are being used, the wavelength channel in terminal nodes passes through the transmitter, switch, multiplexers, demultiplexers, switch and receiver so that the terminal node availability for one wavelength channel and $1: 1$ is

$$
\begin{gathered}
a_{n t, I: I(1)}=a_{T X 1} \times a_{S W 1} \times\left(a_{M U X 1} \cup a_{M U X 2}\right) \times \\
\left(a_{D M U X 1} \cup a_{D M U X 2}\right) \times a_{S W 1} \times a_{R X 1}
\end{gathered}
$$


The availability for pass through nodes is same as for the $1+1$ protection. The availability for all wavelength channels (n) inside a terminal node should be

$$
\begin{aligned}
a_{n t, 1+1(n)}= & a_{T X 1}^{\prime \prime} \times a_{S P L 1}^{\prime \prime} \times\left(a_{M U X 1} \cup a_{M U X 2}\right) \times \\
& \left(a_{D M U X 1} \cup a_{D M U X 2}\right) \times a_{S W 1}^{\prime \prime} \times a_{R X 1}^{\prime \prime}
\end{aligned}
$$

\subsubsection{Node with active components}

An optical switch with add/drop possibilities is used as the active component. Figure 6 represents an optical add/drop multiplexer (OADM) using such an optical switch.

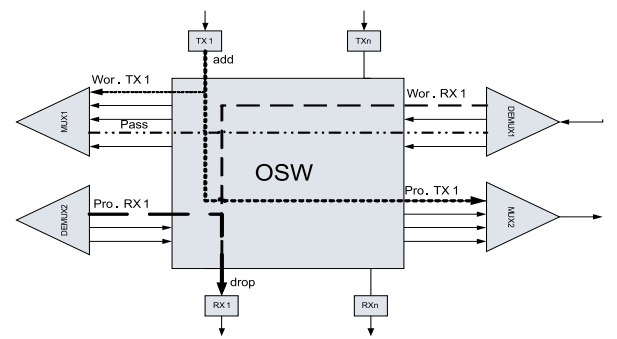

Fig. 6 Node structures with an active optical switch

In the case that the node is used as terminal one, the wavelength channel passes through the transmitter, optical switch, multiplexers, demultiplexers and receiver so the availability for one wavelength channel is

$$
\begin{gathered}
a_{n t,(1)}=a_{T X 1} \times a_{O S W} \times\left(a_{M U X 1} \cup a_{M U X 2}\right) \times \\
\left(a_{D M U X 1} \cup a_{D M U X 2}\right) \times a_{R X 1}
\end{gathered}
$$

The availability for all the wavelength channels in the terminal node should be

$$
\begin{aligned}
a_{n t,(n)}= & a_{T X 1}^{\prime \prime} \times a_{O S W} \times\left(a_{M U X 1} \cup a_{M U X 2}\right) \times \\
& \left(a_{D M U X 1} \cup a_{D M U X 2}\right) \times a_{R X 1}^{\prime \prime}
\end{aligned}
$$

If the node is a pass-through one wavelength channel passes through the multiplexers and optical switch so the availability is

$$
a_{N P}=a_{M U X 1} \times a_{D M U X 1} \times a_{M U X 2} \times a_{D M U X 2} \times a_{O S W}
$$

The availability of terminal nodes is same for the $1+1$ and $1: 1$ protection because the optical switch can act both as the divider and switch.

\section{Availability for WDM ring}

Since the installation of more SDH line systems between two nodes is very expensive, as the capacities of optical cables exhaust considerably, the need for high transmission capacity system requiring only two fibers, has arisen. Such are WDM systems based on wavelength multiplexing which uses the wavelength channel protection. In this section we shall analyse the availability for WDM ring which uses the wavelength channel protection. In this type of protection the switching is carried out on a wavelength channel level so that the protection capacity is one wavelength. There are two types of protection: OCh dedicated protection ring ( $\mathrm{OCh}$ DPRing) and OCh shared protection ring (OCh SPRing).

\subsection{OCh dedicated protection ring}

This type of protection requires two fibers in a ring. Each wavelength channel is being routed on the working path along one side of the ring, and the corresponding dedicated wavelength channel, along the opposite side. Bidirectional wavelength requirements are supported by two wavelength channels; one in each direction. Two types of dedicated protection are possible: $1+1$ and $1: 1$.

In the ring network which uses the $1+1$ wavelength channel protection the wavelength channel in the source node is being duplicated and concurrently delivered in both directions of the ring; working and protection [11].

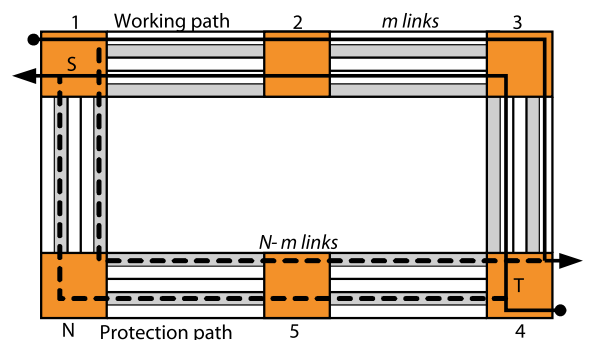

Fig. 7 1+1 wavelength channel protection under conditions without a failure

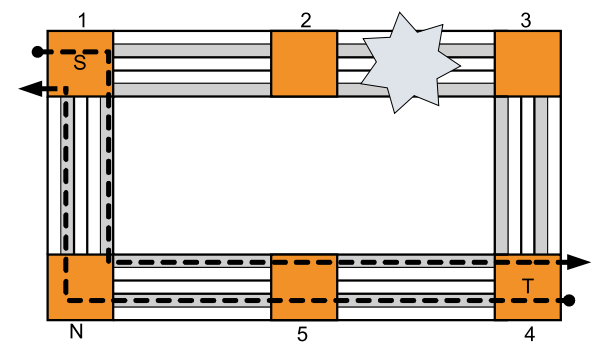

Fig. $81+1$ wavelength channel protection in the case of a failure on working path

Under ordinary conditions in the terminal node, the receivers gets two signal copies (with a different delay) and chooses the best one. In the case of a failure on the working path, the receiver chooses the signal that it gets from the protection path. This is so called single ended protection because the switching is carried out only on one (receiving) side. It is important that the working and protection path do not have common components in order that 
one failure would not cause total communication break down, and this means that the component failures on the working and protection paths are fully independent.

For each wavelength channel of the working path, a corresponding wavelength channel on the protection path is reserved and therefore, we are talking about an $1+1$ wavelength channel protection [8].

Since the wavelength channels on the protection path are persevered we are talking about so called dedicated protection in which no active switch is required. The wavelength channels on the working and protection paths have the same wavelength but that is not a rule. If different wavelengths are being used, the wavelength converters not having big impact on WDM ring availability must be applied.

Let the network generally consist of $\mathrm{N}$ nodes and $\mathrm{N}$ links connecting those nodes [9]. If a wavelength channel on the working path P0 passes through the $m$ number of optical links between terminal nodes, the availability for the working path is equal to the availability product for optical links and nodes through which this wavelength channel passes [10].

$$
\begin{aligned}
a_{s t}\left(P_{0}\right) & =\prod_{i, j \in P_{0}} a_{O L_{i}} \times a_{n_{j}}=\prod_{i \in P_{0}} a_{O L_{i}} \times \prod_{j \in P_{0}} a_{n_{j}} \\
& =\left(a_{n t}\right)^{2} \times\left(a_{n p}\right)^{m-1} \times\left[\prod_{i \in P_{0}}^{m} a_{O L_{i}}\right]
\end{aligned}
$$

In the case of a failure on the working path, the wavelength channel passes the $N-m$ number of optical links and the $N-m-1$ of nodes on the protection path $\mathrm{P} 1$.

The availability for the protection path is [10].

$$
\begin{aligned}
a_{s t}\left(P_{1}\right) & =\prod_{i, j \in P_{1}} a_{O L_{i}} \times a_{n_{j}}=\prod_{i \in R_{1}} a_{O L_{i}} \times \prod_{j \in P_{1}} a_{n_{j}} \\
& =\left(a_{n t}\right)^{2} \times\left(a_{n p}\right)^{N-m-1} \times\left[\prod_{i \in P_{1}}^{m} a_{O L_{i}}\right]
\end{aligned}
$$

The availability for the wavelength channel between the s and $\mathrm{t}$ nodes is completely determined by these two paths so that the availability for the wavelength channel in the case of $1+1$ protection is calculated as the availability of two branches which are fully independent.

$$
A_{s t}(a)=a_{s t}\left(P_{0}\right)+a_{s t}\left(P_{1}\right)-\left[a_{s t}\left(P_{0}\right) \times a_{s t}\left(P_{1}\right)\right]
$$

$$
\begin{aligned}
A_{s t}(a) & =\left(a_{n t}\right)^{2} \times\left(a_{n p}\right)^{m-1} \times\left[\prod_{i \in P_{0}}^{m} a_{O L_{1}}\right]+ \\
& +\left(a_{n t}\right)^{2} \times\left(a_{n p}\right)^{N-m-1} \times\left[\prod_{i \in P_{1}}^{N-m} a_{O L_{i}}\right]- \\
& -\left\{\begin{array}{l}
\left(a_{n t}\right)^{2} \times\left(a_{n p}\right)^{m-1} \times\left[\prod_{i \in P_{0}}^{m} a_{O L_{i}}\right]\left(a_{n t}\right)^{2} \times \\
\times\left(a_{n p}\right)^{N-m-1} \times\left[\prod_{i \in P_{1}}^{N-m} a_{O L_{i}}\right]
\end{array}\right\}
\end{aligned}
$$

where a determines the availability for optical links and nodes. Although in the equation brackets we have the product of the same two members $\left(a_{n t}\right)^{2} \times\left(a_{n t}\right)^{2}$ only one is taken in the expression for availability $\left(a_{n t}\right)^{2}$ because the cause of node failure is the same one.

Therefore we have

$$
A_{s t}(a)=\left(a_{n t}\right)^{2} \times\left\{\begin{array}{l}
\left(a_{n p}\right)^{m-1} \times\left[\prod_{i \in P_{0}}^{m} a_{O L_{i}}\right]+ \\
+\left(a_{n p}\right)^{N-m-1} \times\left[\prod_{i \in R_{i}}^{N-m} a_{O L_{i}}\right]- \\
-\left(a_{n p}\right)^{N-2} \times\left[\prod_{i \in P_{0}, P_{1}}^{N} a_{O L_{i}}\right]
\end{array}\right\}
$$

If we assume that optical links have the same length, their availability is same, i.e

$a_{O L_{i}}=a_{O L}, \forall i$.

In this case, the availability between the $\mathrm{s}$ and $\mathrm{t}$ nodes is

$$
A_{s t}(a)=\left(a_{n t}\right)^{2} \times\left[\begin{array}{l}
\left(a_{n p}\right)^{m-1} \times\left(a_{O L}\right)^{m}+ \\
+\left(a_{n p}\right)^{N-m-1} \times\left(a_{O L}\right)^{N-m}- \\
-\left(a_{n p}\right)^{N-2} \times\left(a_{O L}\right)^{N}
\end{array}\right]
$$

In $1: 1$ protection, the wavelength channel on the front side is not permanently duplicated so that the switching is performed dual-ended which requires a protocol to coordinate it. From the availability aspect, the same availability expression applies as for the $1+1$ protection. The advantage of $1: 1$ protection to the $1+1$ one is that the auxiliary capacity, under conditions without a failure, can be used for the subordinated traffic transmission.

In the case of a failure, the auxiliary capacity is used as the protection one for the superordinated traffic. A disadvantage of 1:1 protection is the complexity because the switching for different OADMs must be synchronous.

\subsection{OCh shared protection ring}

This is the unique type of protection in the ring WDM networks having no equivalent in the SDH ring networks. This protection also uses 2 fibers for connecting the ring nodes. It is actually very similar to an 1:1 protection because it does not have prereserved protection wavelength channels. The difference is that half of the wavelength channels on each fiber is reserved for working traffic and the other half for protection traffic, while in an 1:1 protection all the wavelength channels on a fiber are reserved for the protection, and in a situation without a failure they can be used for the subordinated traffic. Working channels in one fiber are protected by working channels in another fiber. Protection wavelength channels pass along the ring in the opposite direction than the working channels do. Two directions of the bidirectional wavelength requirement are routed on the same side of the ring in opposite fibers. The same wavelength can be reused for some other non-overlapping requirement between different nodes. 


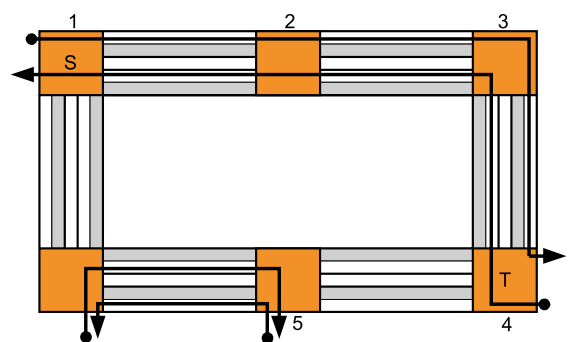

Fig. 9 OCh shared protection under conditions without a failure

When a failure occurs, the damaged wavelength channels are switched in terminal OADMs to another side of the ring and then they use the protection wavelength channels in that fiber. Bidirectional traffic requirements will be routed by using different wavelengths for both directions; otherwise, the wavelength conversion is needed for switching the traffic from working wavelength channel to the protection channel in the opposite direction. In this type of protection there is no prededicated wavelength channels for each wavelength channel of the working path, but there are resources for the damaged wavelength channels. Protection paths will be formed only after a failure on the working path.

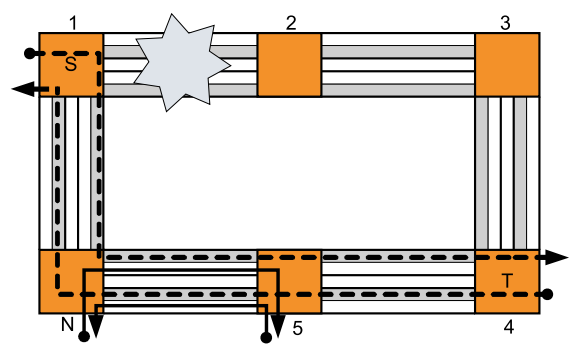

Fig. 10 OCh shared protection under conditions with a failure

When a failure damages all the links between two adjacent OADMs in the ring, the protection path will be established between the source and destination OADM. When a failure occurs, the switching action is required in the source and destination OADM. However, the switching must be carried out for each damaged wavelength. From the availability aspect, the same expression applies for this type of protection as for the $1: 1$ dedicated protection, in that case the capacity in the ring is more efficient.

\section{Numerical results}

As shown before, the same expression applies for the availability of OCh dedicated and OCh shared protection. The only difference is in the nodes structure. For availability calculation we shall use this expression by changing the nodes structure and using different optical link protections: Span Protection and Route Diversity. In order to calculate the availability for a WDM system one needs to know the nodes and optical links availability. For availability calculation, in general, one needs to know the intensity of failures for the individual components. The data are shown in Table 1, and are taken from different sources [9].

NOTE: $w$ is the number of wavelength channels

Availability data for optical components

Tab. 1

\begin{tabular}{|c|c|c|}
\hline Component/Device & Symbol & Failure Rate(FIT) \\
\hline Booster Amplifier & BOA & 3200 \\
\hline Line Amplifier & LOA & 3200 \\
\hline Pre - Amplifier & POA & 3200 \\
\hline Multiplexer & MUX & $25 \mathrm{xW}$ \\
\hline Demultiplexer & DEMUX & $25 \mathrm{xW}$ \\
\hline Optical Switch & OSW & 1000 \\
\hline Fix Transmitter & TRX & 186 \\
\hline Tunable Transmitter & TX & 745 \\
\hline Fix Receiver & RX & 70 \\
\hline Tunable Receiver & RCX & 470 \\
\hline Switch & SW & 50 \\
\hline Splliter & SPL & 50 \\
\hline Cable (per km) & OC & 100 \\
\hline
\end{tabular}

Unavailability and MDT for

Tab. 2 an optical link $\lambda=100 \mathrm{FIT} / \mathrm{km}$

\begin{tabular}{|c|c|c|}
\hline & $\mathrm{U} \times 10^{-6}$ & MDT (min/year) \\
\hline MTTR=12 & & \\
\hline Span Protection & 96.01 & 50.51 \\
\hline Route Diversity & 0.04 & 0.02 \\
\hline MTTR=21 & & \\
\hline Span Protection & 168.01 & 88.30 \\
\hline Route Diversity & 0.13 & 0.07 \\
\hline
\end{tabular}

Optical link unavailability results from the above table are obtained on the assumption that the link length is $80 \mathrm{~km}$ and MTTR $=12$ hours and 21 hours. As it can be seen from Table 2, the unavailability for optical link, as expected, is substantially higher for Span Protection than it is for Route Diversity. Namely, in SP, fibers of the both paths (working and protection) belong to the same cable, so that the same cause of failure actually breaks down the entire communication between the nodes and, therefore, traffic as well. It is also noticeable that the increase in time to repair considerably increases the unavailability. Unavailability for optical nodes with passive and active components was calculated for the instances of 16 and 64 wavelengths, at a time to repair of 4 and 6 hours, which is real time often being taken for the device repair.

As it can be seen from tables 3 and 4, the unavailability for terminal nodes, irrespective of active and passive components, is almost independent on the number of wavelengths. This is logical because the multiplexers are, in fact, in parallel connection for both types of protection (SP and RD). 
Unavailability and MDT (min/year) for a node with passive components

\begin{tabular}{|c|c|c|c|c|}
\hline & \multicolumn{2}{|c|}{$\mathrm{U} \times 10^{-6}$} & \multicolumn{2}{c|}{ MDT } \\
\hline MTTR $=4 \mathrm{~h}$ & $\lambda=16$ & $\lambda=64$ & $\lambda=16$ & $\lambda=64$ \\
\hline Terminal & 1.42 & 1.42 & 0.75 & 0.75 \\
\hline Pass-through & 6.40 & 25.60 & 3.36 & 13.46 \\
\hline MTTR=6 h & & & & \\
\hline Terminal & 2.13 & 2.13 & 1.12 & 1.12 \\
\hline Pass-through & 9.60 & 38.42 & 5.04 & 20.18 \\
\hline
\end{tabular}

Unavailability and MDT (min/year) for

Table 4

a node with active components

\begin{tabular}{|c|c|c|c|c|}
\hline & \multicolumn{2}{|c|}{$\mathrm{U} \times 10^{-6}$} & \multicolumn{2}{c|}{ MDT } \\
\hline MTTR $=4 \mathrm{~h}$ & $\lambda=16$ & $\lambda=64$ & $\lambda=16$ & $\lambda=64$ \\
\hline Terminal & 5.02 & 5.02 & 2.64 & 2.64 \\
\hline Pass-through & 10.40 & 29.60 & 5.47 & 15.56 \\
\hline MTTR=6 h & & & & \\
\hline Terminal & 7.53 & 7.53 & 3.96 & 3.96 \\
\hline Pass-through & 15.60 & 44.40 & 8.20 & 23.34 \\
\hline
\end{tabular}

In pass-through nodes, the increase in the number of wavelengths has an essential impact on the unavailability increase. The reason is a serial connection of the multiplexers (for PC and MTTR $=4$ hours this increase is about 4 times; for AC it is about 2.84 times).

The reason for this different increase is in the fact that in passthrough nodes of AC structure there is an optical switch in serial connection. It can also be seen that the availability for nodes (both terminals and pass-through) with active components, in general, is higher than it is for the same nodes with passive components (for example, for MTTR $=6$ hours, terminal node with active components has 3.5 times higher unavailability than the same node with PC does). The reason is in the fact that an active optical switch has a few times higher probability of failures than the passive switch and divider do. It is also noticeable that the increase in the device time to repair increases unavailability, irrespective of whether the $\mathrm{AC}$ or PC components are concerned, for about 1.5 times regardless of the number of wavelengths.

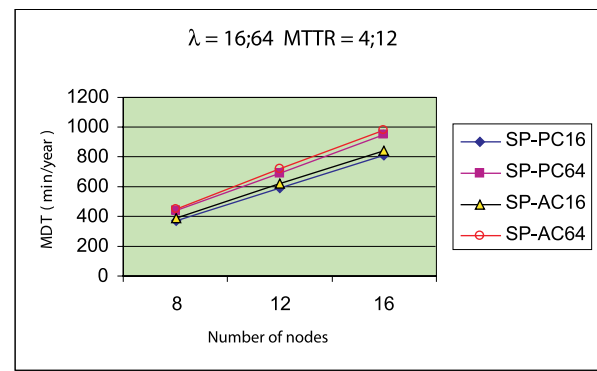

Fig. 13 MDT for Span Protection for MTTR=4; $12 \mathrm{~h}$

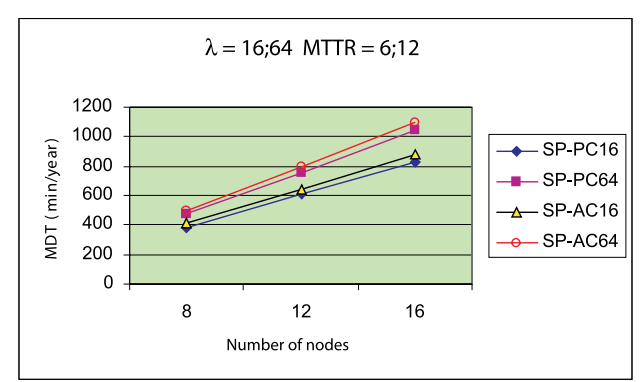

Fig. 14 MDT for Span Protection for MTTR=6; $12 \mathrm{~h}$

We assumed that the ring would consist of 8,12 and 16 nodes; 16 and 64 wavelengths, and that the distance between optical amplifiers and nodes is $80 \mathrm{~km}$. We also analyzed the impact of the reduction in optical link and device time to repair on system availability. It is worst of node that in figures below, during the time to repair (MTTR), the first number refers to the device repair while the other is for the link repair.

In figures 13 and 14 are shown the unavailability and/or MDT for Span Protection with passive and active components, 16 and 64 wavelengths, for various device and optical link times to repair. It is noticeable that the unavailability is almost independent on the number of wavelengths, because the multiplexer in the terminal node is in parallel connection.

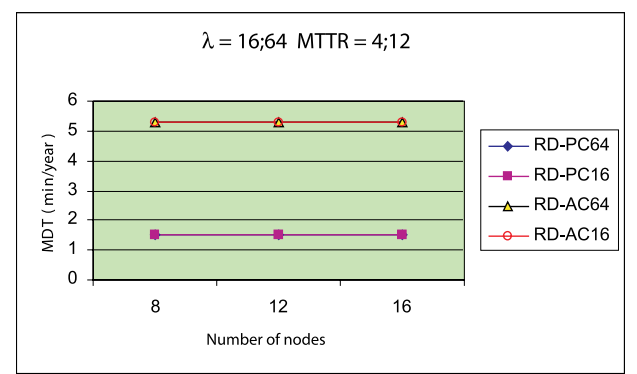

Fig. 15 MDT for Route Diversity for MTTR=4; $12 \mathrm{~h}$

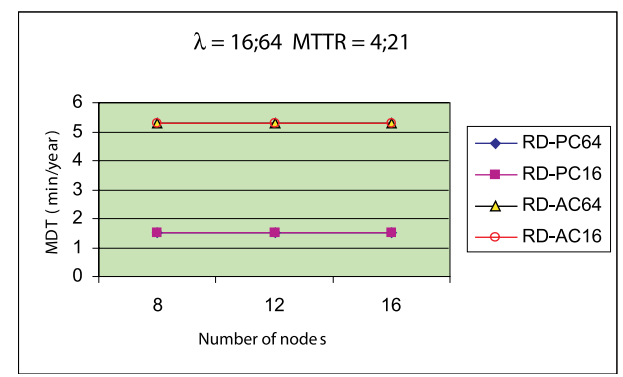

Fig. 16 MDT for Route Diversity for MTTR=4; $21 \mathrm{~h}$

A somewhat higher unavailability is also noticeable if active components are concerned, because there is an optical switch having a significant intensity of failures. As expected too, the unavailability increases by increasing the number of nodes and total distance between terminal nodes, because the impact of optical link 
on the entire unavailability is considerably higher than it is the impact of nodes in this type protection (for example, for $\lambda=16$, $N=12$ the link impact on the entire unavailability for nodes is from $90.25 \%$ to $9.75 \%$ ). In SP, this is an expected result because both the working and the protection path are connected to optical fibers belonging to the same cable. The unavailability increase if optical link time to repair MTTR $=21$, is substantially higher then the MTTR increase for the device. This is expectable because of the significant impact of optical link unavailability on total unavailability.

Figures 15,16 and 17 show the WDM ring unavailability for 8,12 and 16 nodes, which use a Route Diversity protection. It is noticeable that the unavailability for the equal number of nodes and wavelengths is a few dozen times lower than is in SP. The reason is that the working and protection path have no common components (complete separation), so that the breakage of working path optical link does not have the impact in the communication between terminal nodes.

In $\mathrm{RD}$ the impact of nodes on total unavailability is dominant, as expected, because the optical links are completely separated and in parallel connection (for example, for $\lambda=16, N=8$ the link impact on the entire unavailability for nodes is from 3.55 $\%$ to $96.45 \%$ ). It is also noticeable that the number of wavelengths does not have a substantial impact on the entire unavailability either, not it has the number of nodes, and the distance between terminal nodes. Once again, the reason for this is the complete separation of the working and protection path.

It can be seen that the increase in time to repair for nodes has a bigger impact on the entire unavailability than it has the increase in time to repair for optical link (MTTR increase from 4 to 6 hours does increase the unavailability for about $66 \%$ while a MTTR increase of link from 12 to 21 hours does not increase the unavailability at all). The reason for this is again the entire paths independence which means that the one path breakage does not shut down the communication between the nodes.

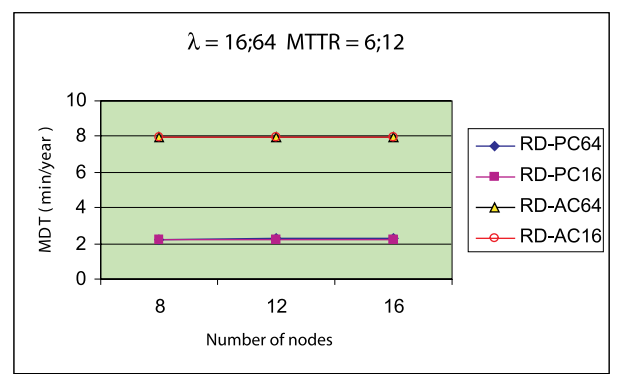

Fig. 17 MDT for Route Diversity for MTTR=6; $12 \mathrm{~h}$

In figures 18 through 21 it is shown the SP and RD unavailability depending on the number of wavelengths and nodes for different times to repair for device and optical link.

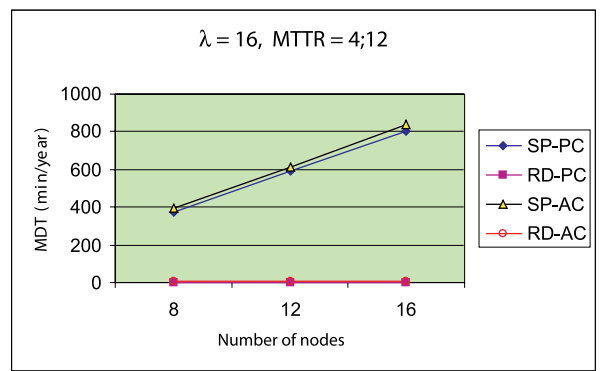

Fig. 18 MDT for $M T T R=4 ; 12 h$ and $\lambda=16$

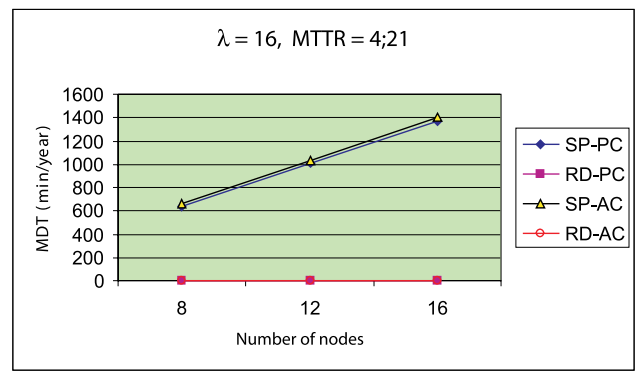

Fig. 19 MDT for $M T T R=4 ; 21 \mathrm{~h}$ and $\lambda=16$

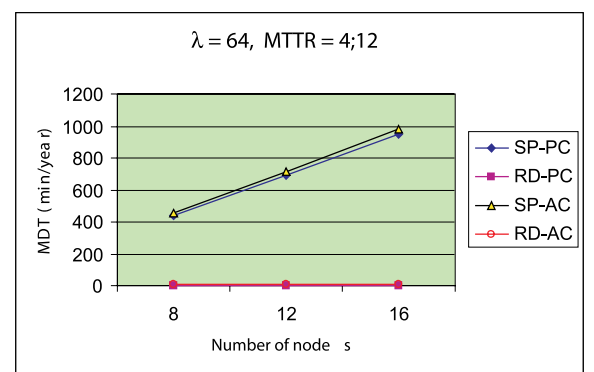

Fig. $20 M D T$ for $M T T R=4 ; 12 h$ and $\lambda=64$

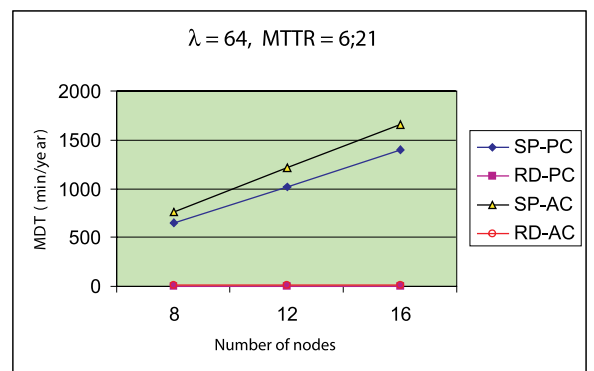

Fig. 21 MDT for $M T T R=6 ; 21 \mathrm{~h}$ and $\lambda=64$

$\mathrm{RD}$, as seen, has a few dozen times lesser unavailability than SP has, for the reasons stated previously. It is also noticeable that in SP, the unavailability increases with the number of nodes, and link lengths, while in RD, that dependence is very small, almost insignificant, because the optical links parallel structure is concerned where a failure in one link does not have the impact on another, so that the communication between the nodes remains retrieved. 
The highest unavailability, as understandable has SP where MTTR $=6 ; 21$ because the optical link impact in this type protection is dominant on the total unavailability. The reason for this it that for optical cable breakage the communication between the terminal nodes is being lost for as long as the very same has not been repaired, because both the working and protection are connected to the fibers of same cable (for $\mathrm{N}=16$ an increase in time to repair from 4 to 12 to 6 and 21 increases the SP unavailability for $59 \%$ ).

Of course, in a WDM ring, irrespective whether SP and RD, the terminal nodes have the biggest impact on the total unavailability because their failure leads to the total communication shut down between those nodes.

\section{Conclusion}

This paper investigates the impact of optical links and node architectures on the availability of connections in WDM ring networks. This work's result analysis shows that Span Protection has substantially higher unavailability than Route Diversity because both directions are connected to the optical links whose fibers belong to the same cable. In RD protection the working and protection paths are completely independent and so such a system is only unavailable in the case of a simultaneous failure on both paths (working and protection) and in the case of a failure in terminal nodes.

By the analysis it is also obtained that the numbers of wavelengths, as well as nodes build-up of active and passive components, do not have a big impact on WDM ring availability, irrespective of the type of protection. It is also established that the impact of optical link time to repair is considerable, while in RD more considerable is the impact of device repair, for the reason that the RD nodes availability has a dominant impact on total unavailability. A WDM ring availability improvement can be achieved by the reduction of time to repair both the optical links and devices. That requires a better maintenance and team organization.

However, a considerable availability improvement can be achieved by the ring networks construction whose working and protection paths have no common components, and they are fully separated, thus offering high availability services to the users.

\section{List of abbreviations}

BOA Booster Optical Amplifier

DPRing Dedication Protection Ring

$\begin{array}{ll}\text { LOA } & \text { Link Optical Amplifier } \\ \text { MTTF } & \text { Mean Time to Failure } \\ \text { MTTR } & \text { Mean Time to Repair } \\ \text { OC } & \text { Optical Cable } \\ \text { OCh } & \text { Optical Channel } \\ \text { OCh DPRing } & \text { Optical Channel Dedication Protection Ring } \\ \text { OCh SPRing } & \text { Optical Channel Share Protection Ring } \\ \text { OPA } & \text { Optical per Amplifier } \\ \text { RX } & \text { Receiver } \\ \text { SPL } & \text { Splitter } \\ \text { SPRing } & \text { Share Protection Ring } \\ \text { SW } & \text { Switch } \\ \text { TX } & \text { Transmitter } \\ \text { 1FIT } & 1 \text { failure in hours }\end{array}$

\section{List of symbols}

$a_{B O A} \quad$ availability of optical booster amplifier

$a_{D M U X} \quad$ availability of demultiplexer

$A_{k} \quad$ Availability of connection

$a_{L O A} \quad$ availability of optical line amplifier

$a_{M U X} \quad$ availability of multiplexer

$a_{n_{j}} \quad$ availability of $\mathrm{j}$-the node which belongs to the working path

$a_{n_{j} p} \quad$ availability of pass-through node

$a_{n p, 1+1(1)}$ availability of pass through node for $1+1$ protection

$a_{n_{j} t} \quad$ availability of terminal node

$a_{n t, 1+1(1)}$ availability of termination node for $1+1$ protection (one wavelength channel)

$a_{n t, 1: 1(1)}$ availability of termination node for $1: 1$ protection (one wavelength channel)

$a_{n t, 1+1(n)}$ availability of termination node for $1+1$ protection (for n wavelength channel)

$a_{O C} \quad$ availability for optical cable

$a_{O L_{i}} \quad$ availability of i-th link on working path

$a_{O L N P_{i}} \quad$ availability of optical link, no protection

$a_{O L, R D} \quad$ availability of optical link, route diversity

$a_{O L, S P} \quad$ availability of optical link, span protection

$a_{O P A} \quad$ availability of optical pre-amplifier

$A_{p} \quad$ Availability of a parallel structure

$a_{R X} \quad$ availability of receiver

$A_{s} \quad$ Availability of a serial structure

$a_{S P L 1} \quad$ availability of splliter

$A_{s t}(a)$ availability for the wavelength channel between the $s$ and $t$ nodes

$A_{s t}\left(P_{0}\right) \quad$ availability of working path between $s$ and $t$ nodes

$A_{s t}\left(P_{1}\right) \quad$ availability of protection path between $s$ and $t$ nodes

$a_{S W} \quad$ availability of switch

$a_{T X 1} \quad$ availability of transmitter

\section{References}

[1] WILlEMS G., ARIJS P., PARYS V. W., DEMEESTER P.: Capacity vs. Availability Trade-offs in Mesh-Restorable WDM Networks, Design of Reliable Communication Networks (DRCN), Proc., Budapest, Hungary, Oct. 2001. 
[2] SCHUPKE A. D.: Reliability Models of WDM Self-Healing Rings, Second International Workshop on the Design of Reliable Communication Networks (DRCN), Munich, Germany, April 9-12, 2000.

[3] JURDANA, I., MIKAC, B.: An Availability Analysis of Optical Cables, Proceedings of WAON'98, pp. 153-160,

[4] INKRET, R.: Shaping of Entirely Optical Transmission Network with Wave Multiplex/masters Degree Thesis, Faculty of Electrical engineering and computing/IT; University in Zagreb, 1998,

[5] INKRET, R.: Availability Modelling of Multi-Service All-Optical Transmission Network, Doctoral Thesis, University of Zagreb, Faculty of Electrical Engineering and Computing, Zagreb, 2004,

[6] ARCI, D., MAIER, G., PATTAVINA, A., PETECCHI, D., TORNATORE, M.: Availability Models for Protection Techniques in WDM Networks, Design of Reliable Communication Networks (DRCN) 2003, Banff, Alberta, Canada, October 19-22, 2003, pp. 158-166,

[7] WILSON, M., R.: The Quantitative Impact of Survivable Network Architecture on Service Availability, IEEE Communications Magazine, May 1998, pp. 122-127,

[8] INKRET, R., MIKAC, B.: Availability Analysis of Different WDM Network Protection Scenarios, Proceedings of WAON'98, pp. 121-128,

[9] RADOS, I.: Influence of Common Path on Availability of the Ring Network, Journal of Telecommunication and Information Technology, National Institute of Telecommunication, Poland, No. 3/2004.

[10] TOMSU, P., SCHMUTZER, C.: Next Generation Optical Networks, PrenticeHall PTR Upper Saddle River, NJ, 2002.

[11] GROVER, W,. D.: Mesh-Based Survivable Networks, Options and Strategies for Optical, MPLS, SONET and ATM Networks, Prentice Hall PTR 2004. 